\title{
Embedded and open star clusters: basic questions
}

\author{
Hans Zinnecker \\ Astrophysikalisches Institut Potsdam, An der Sternwarte 16, D-14482 Potsdam, Germany \\ email: hzinnecker@aip.de
}

\begin{abstract}
This paper provides an introduction to IAU Symposium 266 on star clusters as basic building blocks in space and time. We define clusters as bound systems and discriminate them from general stellar clusterings or groups and unbound associations. We give a few examples of young, embedded, compact clusters which may evolve into looser, open clusters after dynamical relaxation due to mass loss and secular relaxation processes. We ask how and where star clusters form (in normal and interacting galaxies) and provide statistics of open clusters in terms of cluster masses and ages in the solar neighborhood (where observational data are most complete). Finally, we list a number of basic questions for current and future star cluster research and discuss the prospects for cluster studies with the next generation infrared and submillimeter telescopes (Herschel; JWST, E-ELT; ALMA, NOEMA).
\end{abstract}

Keywords. galaxies: star clusters, stars: formation, HıI regions, infrared: stars, X-rays: stars

\section{Introduction}

As the title of this Symposium suggests, star clusters are basic building blocks of galaxies throughout space and time. Ideally, the first question to ask in this context would be how to define a star cluster. I prefer a strict definition for clusters to be gravitationally bound systems, and suggest to discriminate clusters from stellar clusterings or groups and unbound associations. Other immediate questions would be: Where, across the face of galaxies, do star clusters form (mostly in spiral arms?) and do they form continuously or at certain periods in time when galaxies interact (e.g., during close gravitational encounters)? And how did globular clusters form?

As the first speaker at this symposium, I consider it to be my task to whet your appetite for the subject and to raise some of the basic questions in this topical field of research. In an appendix at the end of this paper, I list 12 questions: too many to discuss in this short review, but some of them will be addressed.

The outline of my contribution is as follows. We start by showing and discussing a representative example (NGC 7538), where an evolutionary sequence from dense embedded near-infrared (near-IR) clusters to an optically visible cluster and HII region can be seen in a single, large-scale near-IR image. Then, we briefly describe the emergence of optical open clusters from obscured compact protoclusters. Next, we summarize some of the open cluster statistics, and discuss present-day and initial mass functions of open clusters as well as the distribution of their lifetimes. This then leads to the question as to which fraction of the local field stars has originated from disintegrated open clusters over the course of Galactic history. We also mention the problem of isolated field OB stars and whether they can all be explained as cluster-born runaway stars. We conclude with an outlook at what we can expect from future new IR telescopes such as the JWST and 


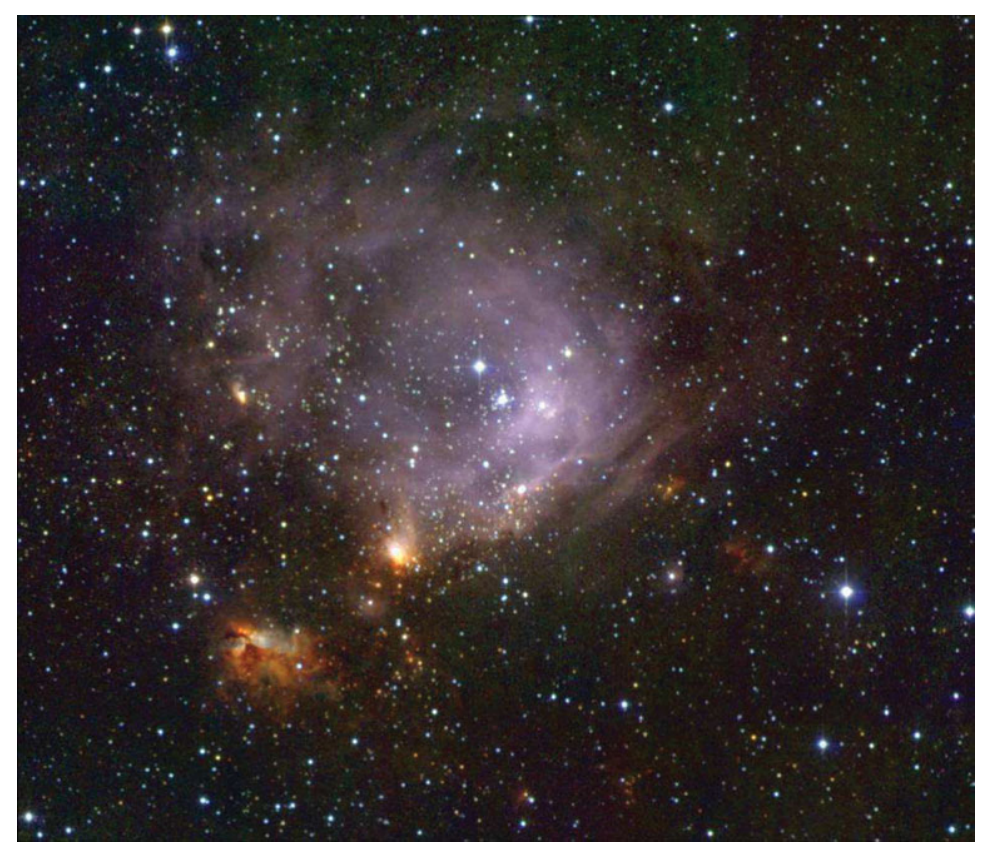

Figure 1. True-color ( $J H K$ ) near-IR image of NGC 7538 (see text for details). (Credit: J. Rayner, M. McCaughrean, H. Zinnecker.)

E-ELT and submillimeter (submm) imaging arrays such as ALMA and NOEMA (the upgraded Plateau de Bure Interferometer, PdBI).

\section{The NGC 7538 HII region: an instructive example}

In Figure 1, we show the HII region NGC 7538, part of the Cas OB2 complex at a distance of $\sim 2.7 \mathrm{kpc}$. This is one of the first large-scale true-color composite infraredarray JHK images (McCaughrean et al. 1991; Zinnecker et al. 1993), and actually made it to the front cover of the August 1991 edition of Sky and Telescope. The image was made by combining three separate mosaics of the region, one each at $J(1.2 \mu \mathrm{m}), H(1.65 \mu \mathrm{m})$, and $K^{\prime}(2.1 \mu \mathrm{m})$. By having these three images represent blue, green, and red respectively, the colors in the resulting composite give some idea of what this region might look like if our eyes were sensitive at near-IR rather than optical wavelengths. In this kind of image, the colors impart real wavelength-dependent information about the various sources. For instance, red objects are cool or embedded in dust, while blue objects are relatively hot.

The image in Figure 1 covers about $12 \times 12 \operatorname{arcmin}^{2}$ (i.e., $\sim 9.5 \times 9.5 \mathrm{pc}^{2}$ at $2.7 \mathrm{kpc}$ ), at a scale of $0.77 \operatorname{arcsec}_{\text {pixel }}{ }^{-1}$. Each of the $J, H$, and $K^{\prime}$ images is a mosaic of 25 overlapping subimages taken, using a NICMOS3 $\mathrm{HgCdTe} 256 \times 256$-pixel array camera on the University of Hawaii 88-inch telescope on Mauna Kea in October 1990. The effective integration time is 2 minutes per pixel per filter, yielding a point-source detection limit of $K^{\prime} \sim 17 \mathrm{mag}$ (or absolute $K$-band magnitude of $\sim 5$, corresponding to young stellar objects with masses as low as $0.3 \mathrm{M}_{\odot}$ at an age of $1 \mathrm{Myr}$ ). The image is more or less centered on the OB stars that illuminate the optical HII region NGC 7538, while the famous IR sources (Werner et al. 1979) are to the south-east. IRS 1, 2, and 3 are in the very bright red core, and IRS 9 is at the apex of the complex reflection nebula. Several new, fainter reflection nebulae are seen, with quite intriguing morphologies. The diffuse HiI-region 
emission seen in purple and pink is probably a combination of thermal Bremsstrahlung and reflected light, both strongest in $J$, along with some ionized hydrogen-line emission in the $K^{\prime}$ filter (Brackett $\gamma$ at $2.166 \mu \mathrm{m}$ ). There are several newly discovered stellar groups in this image, most notably between the HiI region and the compact core hosting IRS $1-3$, and the very red small clusterings to the south, some seemingly aligned in a filamentary substructure. Finally, there are also several dark lanes seen in projection against bright nebulosity, most markedly in the IRS 9 reflection nebulosity.

The overall morphology is of three major condensations, which may form a sequence in age: the diffuse HII region (northwest, oldest), the compact IR core (center), and the less compact IR reflection nebula (southeast, youngest). It is also possible that while star formation has propagated from the HII region to the IRS 1-3 core, the complex to the southeast has evolved independently from the same molecular cloud. The dense cluster between the optical HII region and IRS 1-3 also seems to be evidence for sequential star formation (Elmegreen \& Lada 1977), with the cluster forming from the shock front from the HiI region compressing the surrounding molecular material. The cluster is only revealed now, as the OB stars act to clear away the obscuring gas and dust. A similar case is the NGC 3603 cluster and HII region clearing the view to the NGC 3603-IRS 9 subgroup (see the near- and mid-IR VLT images of Nürnberger 2003); see also the beautiful Spitzer images of the Rosette and RCW 49 clusters. Other examples include the M17 cluster (Hoffmeister et al. 2008; see their JHK VLT image and analysis) and the Sh 2-212 Hit region triggering secondary massive star formation at its border (Deharveng et al. 2008).

Whatever story is revealed by subsequent detailled analysis of these and other images, there is the striking impression that we are seeing different evolutionary stages in a large star-forming-region complex with considerable substructure. Some of the substructure may ultimately merge with the final main cluster, giving rise to an age spread in the cluster stellar population.

While we have emphasized infrared imaging to detect and study young, embedded star clusters, X-ray imaging can also penetrate dust extinction in molecular clouds and reveal embedded populations: the optical depth at $2 \mu \mathrm{m}$ is about the same as at $2 \mathrm{keV}$ ! A splendid example is the Chandra image of the W3 star-forming complex (Feigelson \& Townsley 2008), revealing diverse stellar populations, some of them dense clusters and some more like loose associations.

In conclusion, it is apparent that near-IR and X-ray imaging are capable of revealing a wealth of information, giving us a much clearer view deep inside embedded, dust-obscured stellar nurseries.

\section{From embedded to open clusters}

The example of the NGC 7538 complex above was chosen to illustrate the emergence of an optically visible open cluster and HII region as the end stage of an energetic, internal dynamical evolution. In fact, the birth of optically visible star clusters is reminscent of chicken hatching from their eggs: internal action breaks up the cocoon-like shell. For star clusters, the internal action is due to massive stars, which energize the dense parent cloud with ionizing photons, which in turn generate the gas pressure of an HiI region (with a temperature of about $10000 \mathrm{~K}$ ). The pressure gradient drives the parent gas and dust away from the cluster center and creates a bubble, inside of which the cluster finally becomes transparent to optical radiation (e.g., $\mathrm{H} \alpha$ ). The expansion of the dense gas and dust is assisted by the powerful stellar winds and outflows from the massive OB stars. Note that supernova remnants do not yet play a role at this stage. They only set in 
after some 5-10 Myr have elapsed, but they may be a powerful agent to trigger additional (coeval and coherent) star formation in the wider vicinity of the primary cluster or group, a fine example being the Upper Sco subgroup of the Sco OB2 association (Preibisch \& Zinnecker 2007).

Many embedded star clusters do not survive the expansion of their Hit region, because too much gas and hence binding mass is lost, as first discussed by von Hoerner (1968). In their influential review, Lada \& Lada (2003) argue that only $\sim 10 \%$ of all embedded clusters survive. The rest is immediately released as a field-star population. However, I think the jury is still out as to the true 'infant mortality' by cluster mass fraction. It could be 50 instead of $90 \%$ (still high), in line with the Spitzer data for the Orion A and B molecular clouds, where small groups and clusterings of stars make up about half the total number of young stars, while the rest is found in several big clusters, including the Trapezium (T. Megeath 2009, priv. comm.). Theoretically speaking, the infant-mortality rate depends on the cluster mass and the star-formation efficiency, as described in detail by Parmentier (these proceedings). Those clusters that survive as bound entities may nevertheless lose a fraction of their stars in the process and end up as smaller and less dense clusters. It seems possible that a compact and fairly massive cluster like NGC 3603 may end up as a much looser and lower-mass system, depending on slight changes in the star-formation efficiency and the precise timing of the onset of massive-star-ionization feedback. These speculations can be tested with cluster-formation simulations, where massive stars form last (probably by competitve accretion near the center). We refer to the calcuations by Kroupa et al. (2001), who suggest that the Orion Trapezium cluster may ultimately evolve into a cluster like the Pleiades.

\section{Open cluster statistics: masses and ages}

How do we go about determining the integrated masses of open clusters and from there the present-day and initial mass function (IMF) of open clusters?

There a several methods to infer the masses of star clusters, including direct star counts (lower limits only) or indirect scaling with the stellar IMF (extrapolating the bright end of the IMF to the full IMF, assuming the lower-mass section is known, i.e., assuming a Salpeter or Kroupa-type IMF down to a given lower-mass limit). Dynamical mass determinations are also possible. For example, one can, in principle, use the cluster size and stellar velocity dispersion (hard to obtain in practical terms) to derive virial masses based on the assumption that the cluster is in virial equilibrium (not true for the youngest clusters). This method is frequently used to obtain the masses of molecular clouds in which the star clusters form. Another dynamical method, less well-known, uses tidal masses. At a given location in the Galaxy, the tidal field is known (it can be expressed in terms of Oort constants; cf. King 1962) and hence an observed cluster tidal radius immediately provides the mass enclosed inside this radius. Tidal masses depend on the tidal radius cubed, and the mass accuracy thus depends sensitively on how well the tidal radius can be deduced from King-profile fits of the cluster stellarsurface-density distribution from a limited number of bright member stars. A further caveat is the implicit assumption that the cluster population fills its tidal radius. This is likely true for old clusters but unlikely for very young clusters, which are more compact, with sizes about one tenth the tidal radii (1 pc versus $10 \mathrm{pc}$, roughly speaking). The tidal and the virial method of determining cluster masses are independent of the total cluster luminosity, the latter being the only means to infer extragalactic cluster masses (e.g., Zhang \& Fall 1999; Larsen 2002; de Grijs 2004) using an age-dependent cluster luminosity-mass relation. 
Piskunov et al. (2008) were courageous enough to derive cluster mass functions in the solar neighborhood from a compilation of tidal cluster masses in the range from a few tens to a few hundred thousand solar masses. The data are based on clusters selected from the ASCC-2.5 catalogue, which is complete to apparent integrated cluster $V$-band magnitude of 8 , with 440 clusters (and compact associations) above this completeness limit. The completeness area in the solar neigborhood corresponds to an effective radius of $1 \mathrm{kpc}$ around the Sun. The brightest clusters can be seen far beyond this completeness radius. The local cluster mass function can be fitted with a power-law slope (with an index of -2 ), in agreement with data for extragalactic clusters (derived by converting cluster luminosity into cluster mass functions). The local present-day cluster mass function shows a broad maximum between cluster masses from 30 to $300 \mathrm{M}_{\odot}$.

The sample of open clusters can be binned into several age bins, with as youngest bin that containing clusters of 4-8 Myr. This allowed Piskunov et al. (2008) to derive the IMF of open clusters. The result is a two-segment power law, with a slope of $-5 / 3$ (i.e., different from -2) for the high-mass section above $2000 \mathrm{M}_{\odot}$, while the lower-mass part is characterized by a slope of $-4 / 5$ down to $50 \mathrm{M}_{\odot}$. The interpretation of these results, especially the fact that the initial cluster mass function seems to be different from (flatter than) the overall cluster mass function averaged over all ages, remains to be understood in terms of cluster dynamical evolution. Note that Lada \& Lada (2003) suggested a power-law slope of -2 for their sample of embedded clusters between 50 and $1000 \mathrm{M}_{\odot}$ within 500 pc from the Sun.

Let us end this paragraph with a brief comment about the age determination and distribution of open clusters. The half lifetime for clusters, i.e., the typical open cluster decay timescale, is about $300 \mathrm{Myr}$, again based on the analysis of Piskunov et al. (2006). This is somewhat longer than the original estimate of Wielen (1971). It implies some 30 generations of open star clusters since the formation of the Galactic disk. The surface density of clusters of all ages in the solar neighborhood is about $100 \mathrm{kpc}^{-2}$. The birthrate of young clusters (age bin $4-8 \mathrm{Myr}$ ) is about $0.4 \mathrm{kpc}^{-2} \mathrm{Myr}^{-1}$. Age determination of open clusters is done based on the main-sequence turnoff method. Young clusters can be age-dated using pre-main-sequence tracks and the corresponding $K$-band luminosity function, which shows a feature at the main-sequence turn-on (cf. Zinnecker et al. 1993).

\section{Open clusters as the source of Galactic field stars}

For a long time, the prevailing view has been that about $10 \%$ of all Galactic field stars in the solar neighborhood originated from disintegrating open clusters (Wielen 1971). Recent re-analysis has shown that this fraction is likely as high as 50\% (Piskunov et al. 2008; Zinnecker et al. 2009). The reason for this is that the average mass of young clusters in a statistically complete, magnitude-limited sample is much higher than thought before. Piskunov et al. (2008) estimated the average cluster mass of present-day open clusters of all ages at $700 \mathrm{M}_{\odot}$, while the average initial cluster mass (for clusters after the infantmortality phase of dynamical protocluster gas-mass loss) is $4500 \mathrm{M}_{\odot}$. This shows how much mass open clusters lose during the later stages due to internal and external processes (due to stellar winds and secular stellar dynamical evaporation versus tidal disruption caused by passing molecular clouds; see Gieles, these proceedings).

A related issue is the origin of field OB stars. There appear to be a number of Galactic O stars which do not occur in embedded or open clusters. The question arises to what extent these field $\mathrm{O}$ stars are all runaway stars, either ejected from massive binaries after one star exploded as a supernova or ejected from dense clusters due to internal dynamical slingshot interactions involving transfer of potential energy from multiple systems into 
kinetic energy several times the escape speed from the parent cluster. A recent study by Schilbach \& Röser (2008) concluded that most O-type runaway stars with good propermotion determinations and radial velocities can be traced back directly to some young parent cluster in a Galactic potential. A similar study for B-type runaway stars is not yet available. The existence of randomly located field OB stars is important for widely distributed effective supernova energy input into the interstellar medium as a source of turbulence.

\section{Future prospects with next-generation telescopes}

Let us try to cast an eye at the future of young star cluster research, both groundbased and space-borne. The ALMA submm array in Chile will be operational in 2012 and from that point will give us detailed high-angular-resolution images of protocluster clouds, at various evolutionary stages. It will resolve cold, dense protostellar clumps destined to become stars. A glimpse can already be gleaned from the results of current millimeter interferometers such as CARMA, SMA, and $P d B I$ (the latter will be upgraded from 6 to 12 telescopes to become the Northern Extended Millimeter Array, NOEMA, in the near future). The Herschel far-IR satellite observatory with its PACS and SPIRE imaging cameras, now in operation, will soon provide us with clump-mass distributions and fragmentation patterns in protoclusters and offer us clues as to the origin of the stellar IMF in clusters (Ph. André 2009, priv. comm.).

Further down the line, JWST-because of its extreme sensitivity in the thermal infrared-will likely spatially resolve not only the luminous, but also the faint energy sources in nearby protoclusters and protostellar groups, shedding light on massive-star-formation processes, such as in the Kleinmann-Low nebula (IRC 2) behind the Orion Nebula Cluster. It is still unclear what is going on in this nearest but most heavily obscured site of massive star formation. JWST (both with NIRCAM and MIRI) may also be instrumental to address the question of initial mass segregation in protoclusters, as it can map the location of massive protostellar objects with respect to their lower-masss siblings at very early, very deeply embedded stages before dynamical evolution can change the birth configuration. Recent studies of young infrared star clusters suggested there is no evidence for mass segregation (Ascenso et al. 2008), contrary to expectation based on the model of competitive accretion (Bonnell et al. 2007). The ultimate facility to study the dense centers of massive star clusters will be the E-ELT (first light expected around 2018). Adaptive-optics observations with a $42 \mathrm{~m}$ diameter telescope in the near-IR $K$ band $(2 \mu \mathrm{m})$ will go a long way to penetrate even 100 magnitudes or more of visual extinction and resolve even very compact clusters, in both imaging and velocity space, in distant Galactic star-forming regions, including the massive Sgr B2 millimeter continuum cores near the Galactic Centre at $8 \mathrm{kpc}$ where analogs of the Arches or Quintuplet massive clusters may be forming now (J. Bally 2009, priv. comm.). The science case for E-ELT near/thermal-IR and integral-field spectroscopy is further described in the Proceedings of IAU Symposium 232 (Zinnecker 2006).

\section{Appendix: 12 questions}

- Definition of a 'cluster' (bound versus unbound, clusterings or groups);

- Origin of the cluster mode of star formation versus distributed mode;

- Statistics of embedded clusters in the solar neighborhood (1-2 kpc);

- 'Infant mortality' (dynamical evolution during early gas-mass loss);

- Open clusters as survivors (NGC 3603 precursor of the Pleiades?); 
- Determination of open cluster masses, ages, and lifetimes (methods);

- Statistics of open clusters in the solar neighborhood (masses, ages);

- What fraction of field stars originated in open clusters? binaries?

- OB clusters versus OB associations: an evolutionary sequence or not?

- Do all massive stars form in clusters? what about runaway OB stars?

- Progenitors of massive Galactic starburst (Wolf-Rayet/red-supergiant) clusters;

- Origin of Population II globular clusters (minimum metallicity $Z=0.01 \mathrm{Z}_{\odot}$ ?).

\section{Acknowledgements}

I thank Richard de Grijs for the invitation to present this introductory contribution, Charles Lada and Anatoly Piskunov for insightful discussions, and the German DAAD for providing travel funds to participate in the IAU General Assembly in Rio de Janeiro and this particular IAU Symposium.

\section{References}

Ascenso, J., Alves, J., \& Lago, M. T. V. T. 2009, A\& A, 495, 147

Bonnell, I. A., Larson, R. B., \& Zinnecker, H. 2007, in: B. Reipurth, D. Jewitt \& K. Keil (eds.), Protostars and Planets $V$ (Tucson: University of Arizona Press), p. 147

de Grijs, R. 2004, in: H. J. G. L. M. Lamers, L. J. Smith \& A. Nota (eds.), The Formation and Evolution of Massive Young Star Clusters, ASP Conf. Ser. (San Francisco: ASP), Vol. 322, p. 29

Deharveng, L., Lefloch, B., Kurtz, S., Nadeau, D., Pomarès, M., Caplan, J., \& Zavagno, A. 2008, $A \mathscr{E} A, 482,585$

Elmegreen, B. G. \& Lada, C. J. 1977, ApJ, 214, 725

Feigelson, E. D. \& Townsley, L. K. 2008, ApJ, 673, 354

Hoffmeister, V. H., Chini, R., Scheyda, C. M., Schulze, D., Watermann, R., Nürnberger, D., \& Vogt, N. 2008, ApJ, 686, 310

King, I. 1962, $A J, 67,471$

Kroupa, P., Aarseth, S., \& Hurley, J. 2001, MNRAS, 321, 699

Lada, C. J. \& Lada, E. A. 2003, ARA $\& A, 41,57$

Larsen, S. S. 2002, $A J, 124,1393$

McCaughrean, M. J., Rayner, J. T., \& Zinnecker, H. 1991, MemSAIt, 62, 715

Nürnberger, D. 2003, A\& $A, 404,255$

Piskunov, A. E., Kharchenko, N. V., Röser, S., Schilbach, E., \& Scholz, R.-D. 2006, A\&\&A, 445, 545

Piskunov, A. E., Kharchenko, N. V., Schilbach, E., Röser, S., Scholz, R.-D., \& Zinnecker, H. 2008, A\& $A, 487,557$

Preibisch, T. \& Zinnecker, H. 2007, in: B. G. Elmegreen \& J. Palous (eds.), Triggered Star Formation in a Turbulent ISM, IAU Symp. 237, p. 270

Schilbach, E. \& Röser, S. 2008, A\& $A$, 489, 105

von Hoerner, S. 1968, in: Y. Terzian (ed.), Interstellar Ionized Hydrogen (New York: Benjamin), p. 101

Werner, M. W., Becklin, E. E., Gatley, I., Matthews, K., Neugebauer, G., \& Wynn-Williams, C. G. $1979, M N R A S, 188,463$

Wielen, R. 1971, A\& A, 13, 309

Zhang, Q. \& Fall, S. M. 1999, ApJ (Letters), 527, L81

Zinnecker, H. 2006, in: P. Whitelock, M. Dennefeld \& B. Leibundgut (eds.), The Scientific Requirements for Extremely Large Telescopes, IAU Symp. 232, p. 324

Zinnecker, H. 2009, in: J. Andersen, J. Bland-Hawthorn \& B. Nordstroem (eds.) The Galaxy Disk in Cosmological Context, IAU Symp. 254, p. 221

Zinnecker, H., McCaughrean, M. J., \& Wilking, B. A. 1993, in: E. H. Levy \& J. I. Lunine (eds.), Protostars and Planets III (Tucson: University of Arizona Press), p. 429 